\title{
DESIGN ALTERNATIVES OF NETWORK OF ALTMANN LINKAGES
}

\author{
F. ATARER ${ }^{1}$, K. KORKMAZ ${ }^{1} \&$ G. KIPER ${ }^{2}$ \\ ${ }^{1}$ Department of Architecture, Izmir Institute of Technology, Gülbahçe, 35430, Izmir, Turkey. \\ ${ }^{2}$ Department of Mechanical Engineering, Izmir Institute of Technology, Gülbahçe, 35430, Izmir, Turkey.
}

\begin{abstract}
This paper presents a method of building deployable network assemblies derived from the single degree of freedom (DoF) over constrained Altmann linkage as a basic module. The method is based on assembling linkages with common links and joints or overlapping with extra $\mathrm{R}$ or $2 \mathrm{R}$ joints. New loops are emerged with overlapping method. The networks created have a single DoF, are over-constrained and have both fully deployed and folded configurations. The computer-aided models (CAD) are used to demonstrate these derived novel mechanisms.

Keywords: Altmann linkage, multi-loop deployable mechanism, over-constrained linkage.
\end{abstract}

\section{INTRODUCTION}

Recently, many single-loop overconstrained linkages have been used as modules in deployable mechanisms. Overconstrained linkages have full-range mobility even though they do not meet the Grübler-Kutzbach mobility criterion [1]. Modular single degree of freedom (DoF) deployable structures are obtained by assembling several identical modules, which are usually single DoF loops. In the literature, significant single loop overconstrained linkages such as Bennett, Myard, Sarrus and Bricard linkages have been used as modules for deployable structures.

The Bennett linkage [2] is a well-known spatial closed loop 4R ( $R$ : revolute joint) linkage of four links having the axes of $\mathrm{R}$ joints neither parallel nor concurrent. Baker and $\mathrm{Hu}$ [3] attempted to connect two similar Bennett linkages together with only $\mathrm{R}$ joints but they obtained rigid structures. Chen's studies [4] illustrate two different methods for derivation of network of Bennett linkages. These are single-layer and multi-layer networks. Using single-layer network of Bennett linkages, Chen obtains a flat deployable structure that expands to form a deployable arch. Additionally, using multi-layer network of Bennett linkages, a curved form is obtained. After that, Melin [5] developed a new type of deployable shelter based on tiled Bennett mechanisms. The shelter deploys into a half-cylinder shape.

The Myard 5R linkages [6] are closed loops of five links. These linkages are obtained by assembling two Bennett linkages with some common links/joints and then removing some of the common links/joints. Huang's studies [7] show three different connections of Myard linkages that are central, peripheral and both central and peripheral connections.

The Sarrus linkage [8] is the first spatial overconstrained linkage that has ever been published. It is a 6R linkage where the links are hinged with two sets of three parallel hinges and the directions of these two sets are different. Calatrava [9] derived various deployable networks with Sarrus linkage as a basic module. Bouten [10] presented flat and polar assemblies of Sarrus arrays. Kiper and Söylemez [11] presented deployable polyhedral linkages comprising Sarrus loops.

Bricard [12] discovered and reported six distinct types of mobile 6R linkages that are the general line-symmetric case, the general plane-symmetric case, the trihedral case, the line-symmetric octahedral case, the plane-symmetric octahedral case and the doubly 
collapsible octahedral case. Chen [4] introduced hybrid Bricard linkages that deploy from a compact folded configuration to a flat triangular expanded configuration. Chen obtained flat array of Bricard linkages whose shape is like a star. Huang et al. [13] proposed a new family of single DoF deployable networks using the threefold-symmetric Bricard mechanisms. Cui et al. [14] used general line-symmetric Bricard linkages to assemble deployable antennas.

This study examines the possibility of building network of single DoF deployable structures using an Altmann linkage as a basic module. It explores the probability of assembly alternatives with only $\mathrm{R}$ joints.

This paper consists of four sections. Section 2 comprises the description of the Altmann linkage. Section 3 is concerned with network of Altmann linkages and its derivation types. In this section, the mobility of each network is calculated and CAD models of them are presented. In Section 4, a summary of what has been achieved is given.

\section{ALTMANN LINKAGE}

The Altmann linkage [15] is a single DoF single loop overconstrained 6R linkage. It is a special case of Bricard's line-symmetric linkage. Fig. 1a shows a construction of the Altmann linkage. Each $\mathrm{R}$ joint axis is perpendicular to the adjacent joint axis as is seen in Fig. $1 \mathrm{~b}$.

The Denavit-Hartenberg parameters of the Altmann linkage are expressed as follows [16]:

$$
\begin{array}{lll}
a_{12}=a_{45}=a & a_{23}=a_{56}=0 & a_{34}=a_{61}=b \\
a_{12}=a_{45}=\frac{\pi}{2} & a_{23}=a_{56}=\frac{\pi}{2} & a_{34}=a_{61}=\frac{3 \pi}{2}
\end{array}
$$

The DoF of a mechanism according to the Grübler-Kutzbach mobility criterion [1] is calculated as:

$$
M=\lambda(n-1)-\sum_{i=1}^{5}(\lambda-i) p_{i}
$$

where $\lambda$ is the DoF of space in which the mechanism operates ( $\lambda=6$ for spatial mechanisms), $\mathrm{n}$ is number of links and $\mathrm{p}_{\mathrm{i}}$ is number of joints having i DoFs. According to the above equation, mobility of the Altmann linkage for $\lambda=6$ can be calculated as follows.

$$
M=6(6-1)-5 \times 6=0
$$

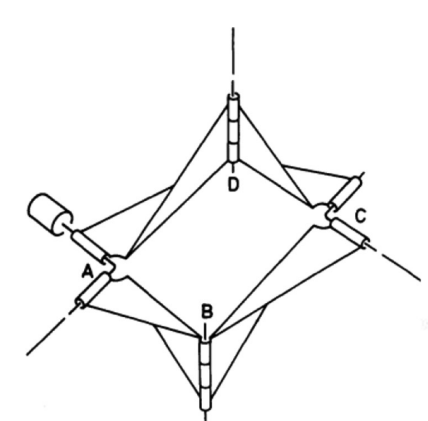

(a)

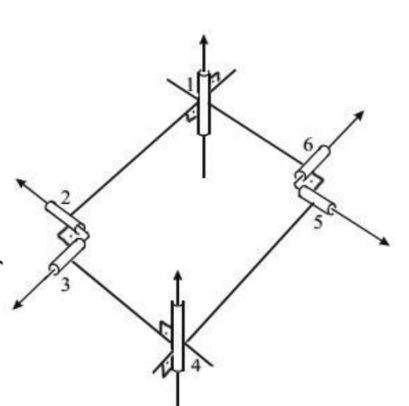

(b)

Figure 1: Altmann Linkage and its 3D schematic diagrams [16]. 
Thus, the formula fails to predict the mobility of the Altmann linkage. Even though Altmann linkage does not meet the mobility criterion, it has full-range mobility. Altmann linkage is an exception like all other overconstrained mechanisms because of its special geometric conditions among the joint axes. Actually, the Altmann linkage operates in $\lambda=5$ space. The formula (3) for subspace $\lambda=5$ gives the correct DoF:

$$
M=5(n-1)-4 p_{1}=5(6-1)-4 \times 6=1
$$

\section{NETWORK OF ALTMANN LINKAGES}

Deployable networks are usually derived from a set of basic deployable modules. With this derivation, one can assemble a set of deployable modules into multi-loop deployable networks without changing the mobility.

In Fig. 2, a 2D schematic diagram of basic module of Altmann linkage is drawn such that two pairs of links $a$ and $b$ are represented by thick black lines and two links $c$ are represented by black squares. Four R joints 2, 3, 5, 6 connecting the link $c$ to links $a$ and $b$ are represented by thin black lines, while two R joints 1,4 connecting links $a$ and $b$ are represented by black circles. This schematic representation is used for presenting the derivation process of the networks of Altmann linkages.

Two alternative ways of connections are proposed to build the networks. The first type is connection with common links or joints and the second one is connection with new joints. This section examines the variations for the deployable network of the Altmann linkages via examples.

\subsection{Network of Altmann linkages with common links and joints}

This network includes three different types. The first type is the scissor version that moves like a scissor linkage; the second one is the arch version and the third one is the dome version. They are multi-loop spatial mechanisms consisting of at least two Altmann linkages. The mobility of the networks is calculated according to the mobility formula proposed by Alizade [17] for multi-loop mechanisms:

$$
M=\sum_{i=1}^{j} f i-\sum_{k=1}^{L} \lambda_{k}+q
$$

where $j$ is the number of joints, $f_{i}$ is DoF of the $\mathrm{i}^{\text {th }}$ joint, $L$ is the number of independent loops, $\lambda$ is the $D o F$ of space in which the mechanism operates $(\lambda=2,3,4,5,6)$ and $q$ is the number of excessive elements (link, joints or loops).
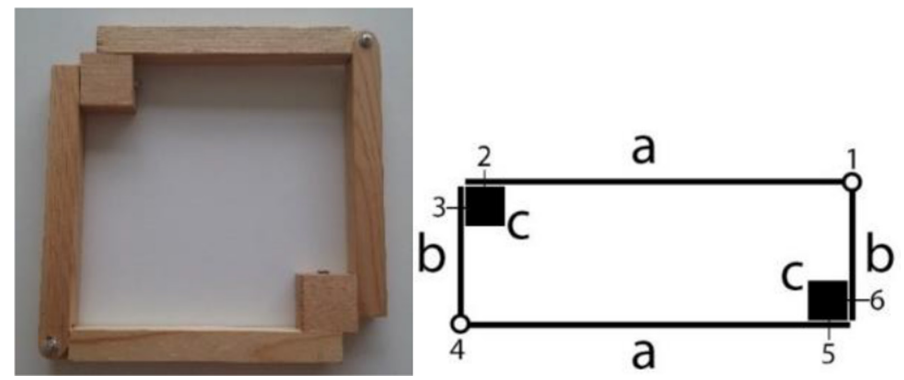

Figure 2: Model and schematic diagram of basic Altmann module. 


\subsubsection{Type-1: Scissor version}

Planar scissor-like linkage is well-known module used for deployable structures. By connecting two Altmann linkages, a spatial scissor mechanism is obtained with two common links and a common joint as seen in Fig. 3. Common links $(a+b)$ and common joint $(R)$ are represented with red color. The network works like a scissor mechanism. Although two more loops are assembled, the mobility does not change. There are 18 links, 21 (R) joints and 4 loops operate in $\lambda=5$ space as seen in Fig. 3 . There are no excessive elements, so according to eqn (6):

$$
M=\sum_{i=1}^{j} f i-\sum_{k=1}^{L} \lambda_{k}+q=21-5 x 4=1
$$

Any number of new loops can be added to the network. In this network, every new additional module does not alter the mobility and the foldability of the network. Therefore, it can be easily moved from deployed to folded configuration without any errors. Fig. 4 illustrates CAD model of deployed, semi deployed and folded configurations.

\subsubsection{Type-2: Arch version}

Arch version is obtained by connecting two Altmann linkages with two common links and a common joint as seen in Fig. 5. Common links $b, c$ and common joints are represented with red color. After the assembling proc ess in the flat form, it can be easily reduced in size to be a compact bundle or deployed to form an arch as in Fig. 6. More Altmann linkages can be assembled to the network without altering the mobility. In Fig. 6, there are 15 (R) joints, 3 (2R) joints and 4 loops operate in $\lambda=5$ space. According to eqn (6):

$$
M=\sum_{i=1}^{j} f i-\sum_{k=1}^{L} \lambda_{k}+q=21-5 \times 4=1
$$
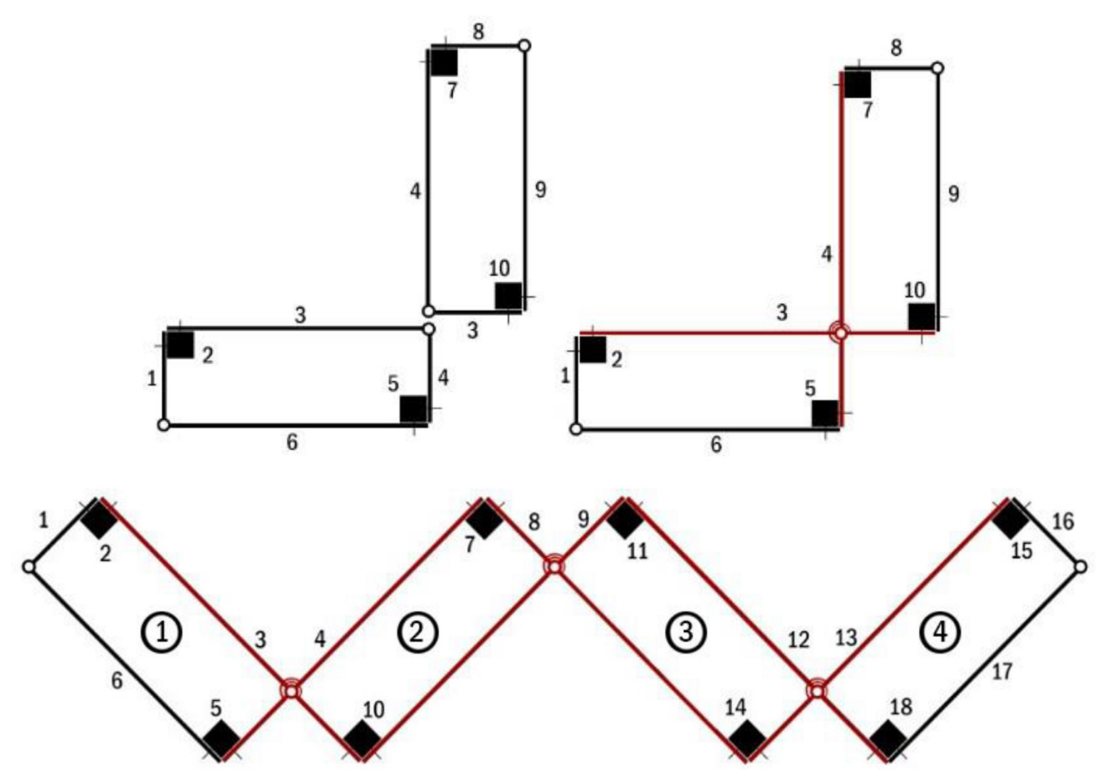

Figure 3: Schematic diagram of network of scissor version. 


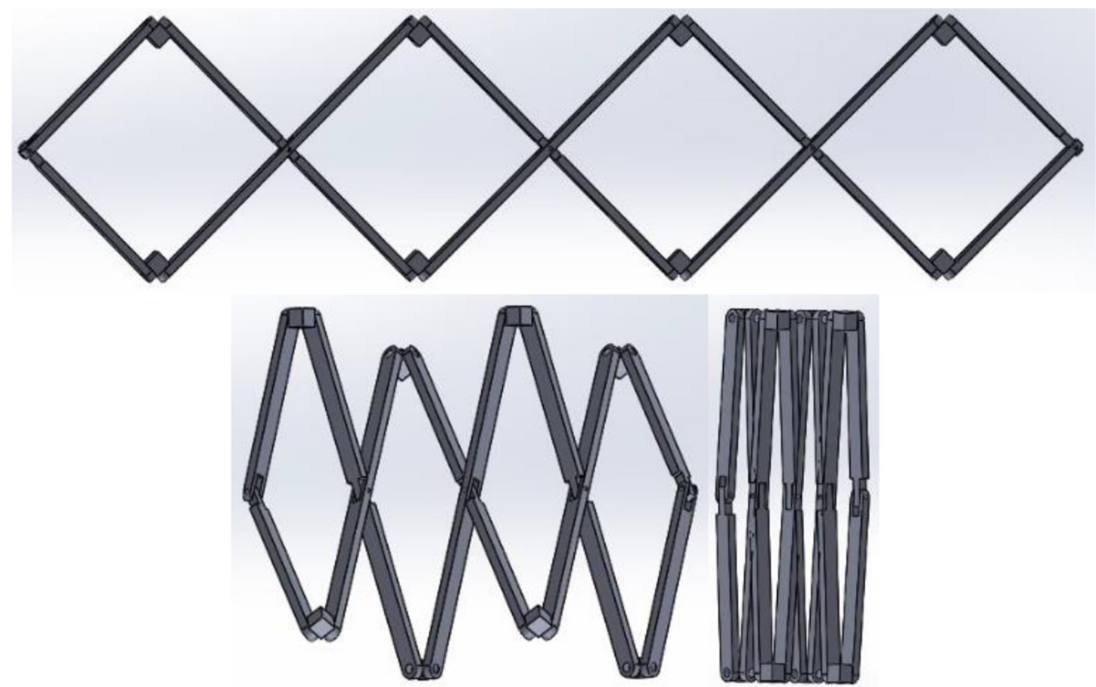

Figure 4: Deployment stages (deployed, semi-deployed and folded) of scissor version.

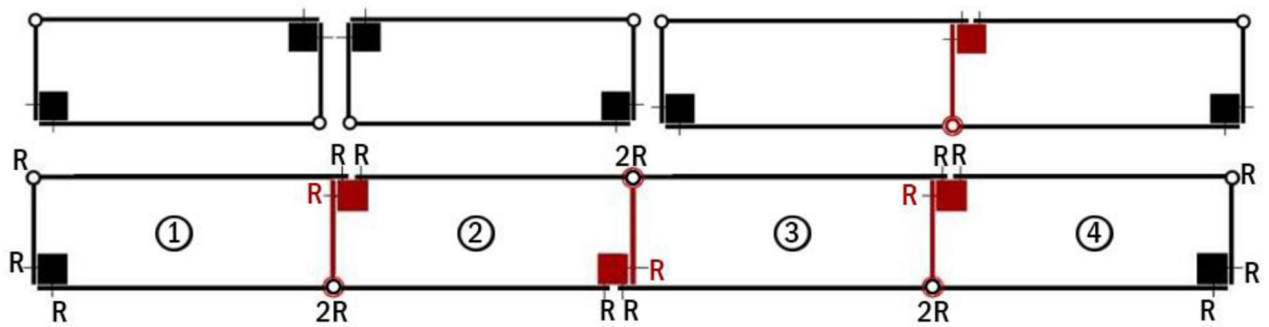

Figure 5: Schematic diagram of arch version.

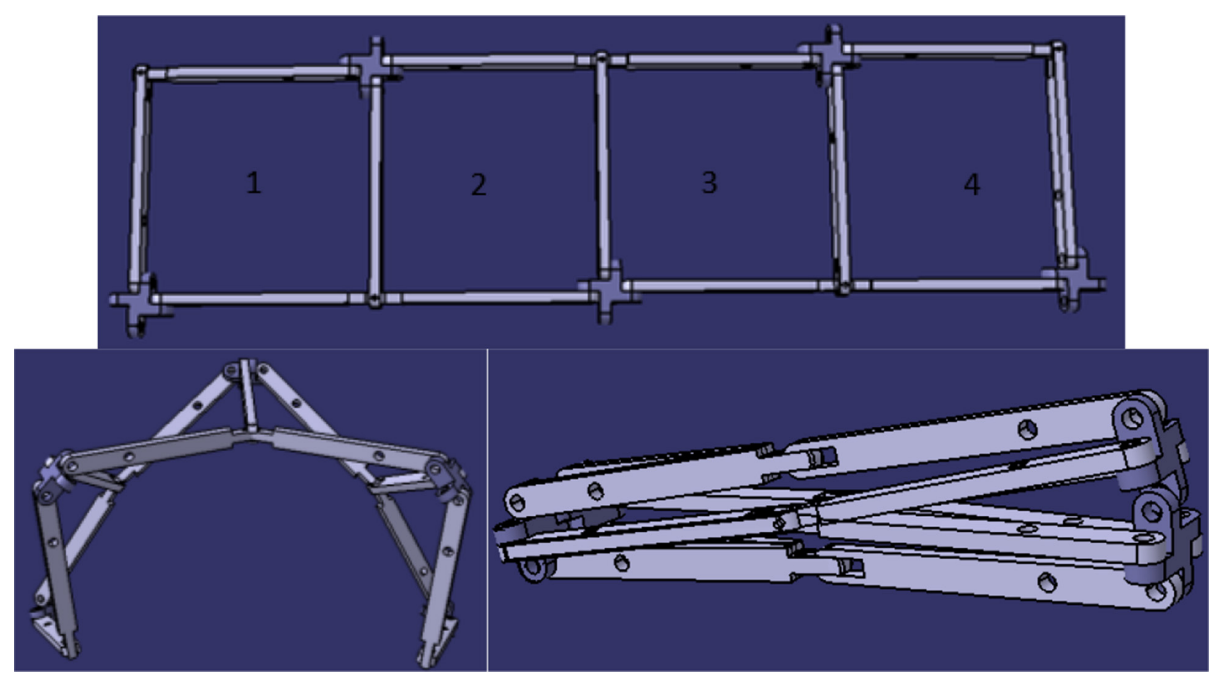

Figure 6: Deployment stages (deployed, semi-deployed and folded) of arch version. 


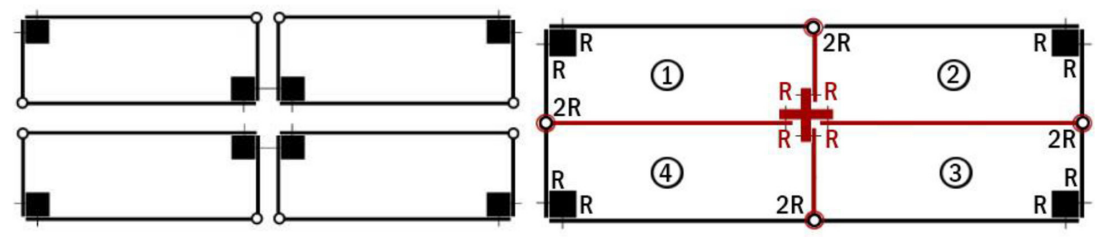

Figure 7: Schematic diagram of dome version.

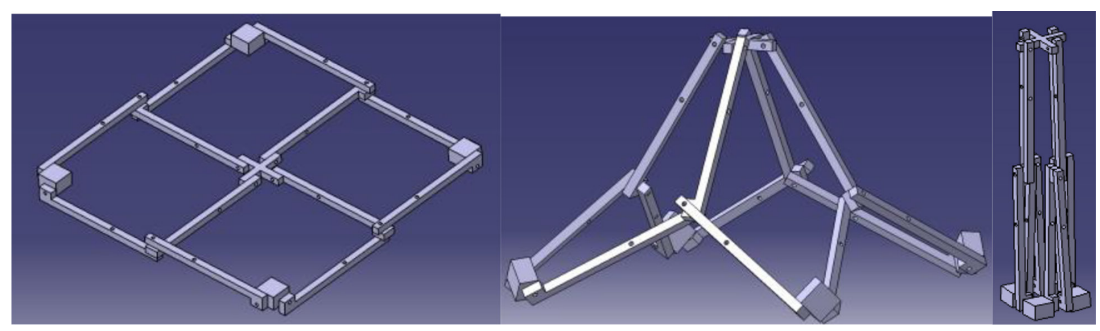

Figure 8: Deployment stages (deployed, semi-deployed and folded) of the dome version.

\subsubsection{Type-3: Dome version}

A single DoF dome version is obtained by connecting four Altmann linkages with two common links and a common joint between adjacent Altmann loops as seen in Fig. 7. Common links and common joints are represented with red color. This network consists of $12(\mathrm{R})$ joints, 4 (2R) joints and 4 loops operate in $\lambda=5$ space. In this case, $q$ is non-zero and it is the number of dependent loops. When three or more loops are tiled around a center, one of them is dependent. Let's say here loop number 4 is dependent. Thus $q$ is equal to 1 . According to eqn (6):

Unlike the two previous types, it is not possible to add more loops to the dome type. Figure 8 illustrates CAD model of deployed, semi-deployed and folded configurations. In this network, a hub is set in order to connect four Altmann basic modules around it.

\subsection{Network of Altmann linkages with new joints}

The second method for obtaining networks includes two different types. The first type is Altmann loop version that involves small Altmann loops and second one is parallelogram version that involves small parallelogram loops. They are multi-loop spatial mechanisms consisting of at least two Altmann loops and one small extra loop. The mobility of the networks is calculated according to the mobility formula (6) for multi-loop mechanisms.

\subsubsection{Type-1: Altmann loop version}

Altmann loop version is obtained by overlapping two basic Altman modules with two new $2 \mathrm{R}$ joints as is seen in Fig. 9-10. In this type, a new loop $(\lambda=5)$ is emerged. The network of the Altmann loop version is obtained with four linkages connected with six new $2 \mathrm{R}$ joints represented with red circles. Three new loops are emerged. The network consists of $24(\mathrm{R})$ joints, $6(2 \mathrm{R})$ joints and 7 loops operate in $\lambda=5$ space. According to eqn (6):

$$
M=\sum_{i=1}^{j} f i-\sum_{k=1}^{L} \lambda_{k}=36-(5 \times 7)=1
$$




\subsubsection{Type-2: Parallelogram loop version}

Parallelogram loop version is obtained by overlapping two basic Altmann linkages with two new $\mathrm{R}$ joints as is seen in Fig. 11. In this type, a new loop $(\lambda=3)$ is emerged. The network of Parallelogram Loop version is obtained with four linkages connected with six new $\mathrm{R}$ joints represented with red circles. Three new loops are emerged. The network consists of $30(\mathrm{R})$ joints and 7 loops. Loops numbered 1, 3, 5, 7 operate in $\lambda=5$ space and loops 2, 4, 6 operate in $\lambda=3$ space. According to eqn (6):

$$
M=\sum_{i=1}^{j} f i-\sum_{k=1}^{L} \lambda_{k}=30-(5 \times 4)+(3 \times 3)=1
$$
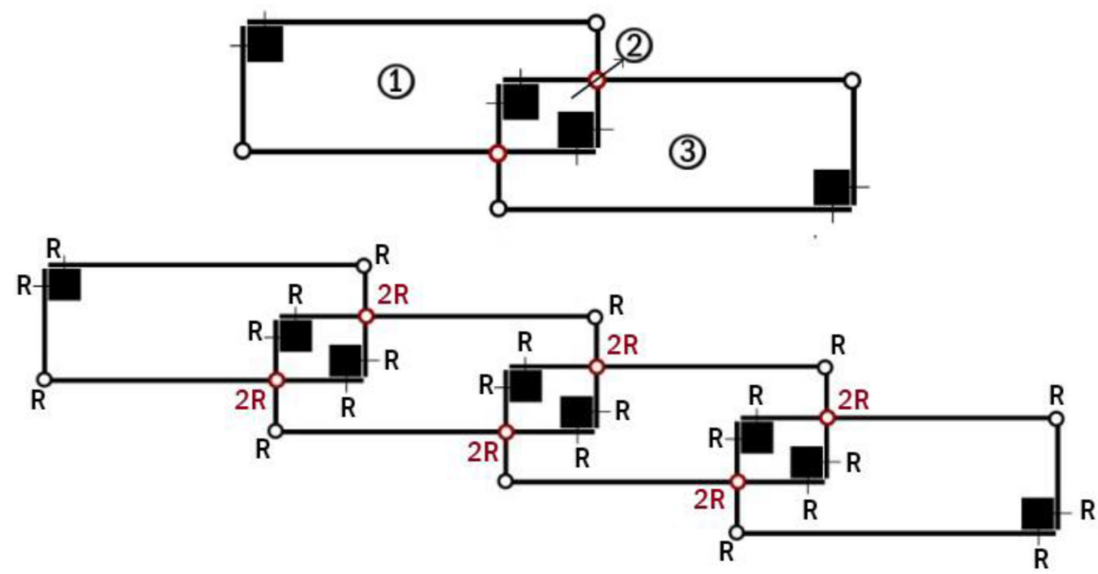

Figure 9: Schematic diagram of multi-loop deployable module of Altmann loop version.

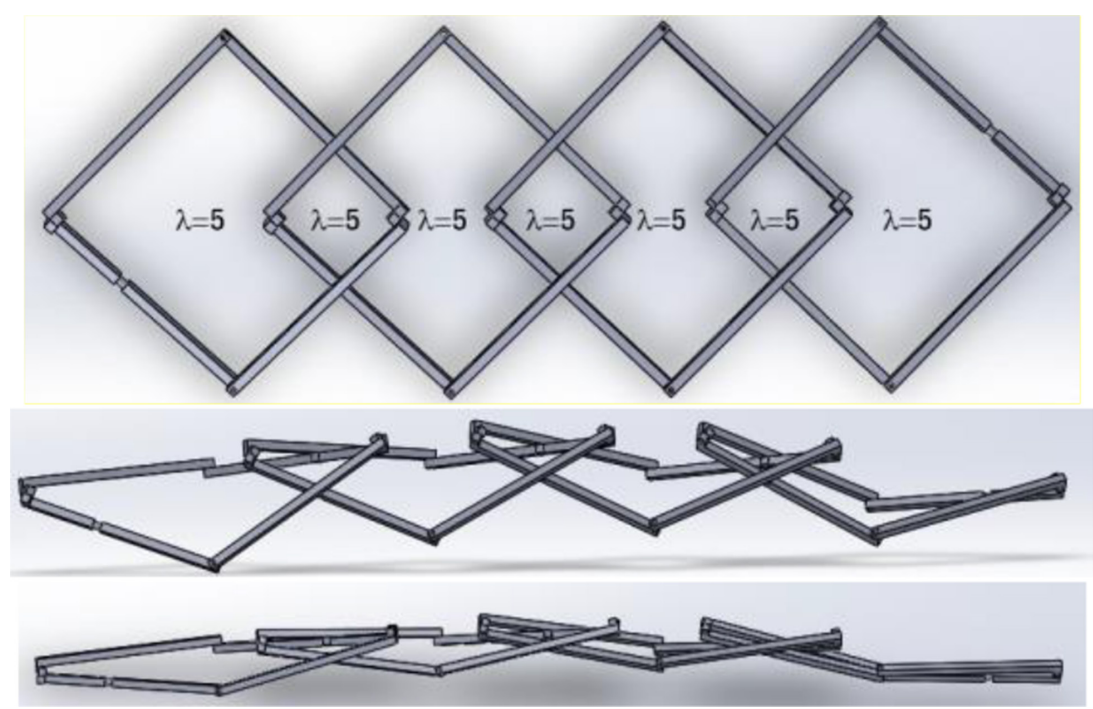

Figure 10: Deployment stages (deployed, semi-deployed and folded) of Altmann loop version. 


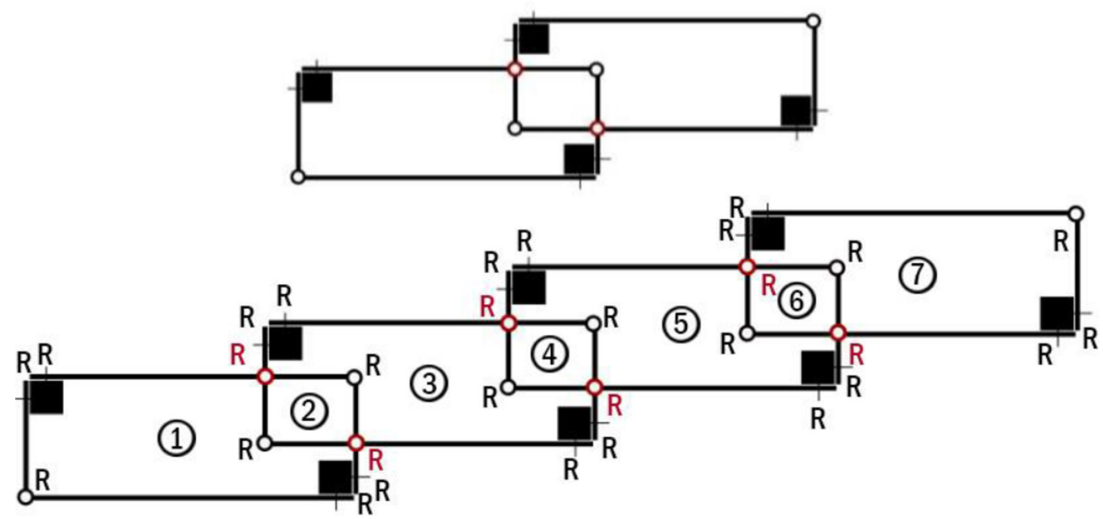

Figure 11: Schematic diagram of multi-loop deployable module of parallelogram loop version.

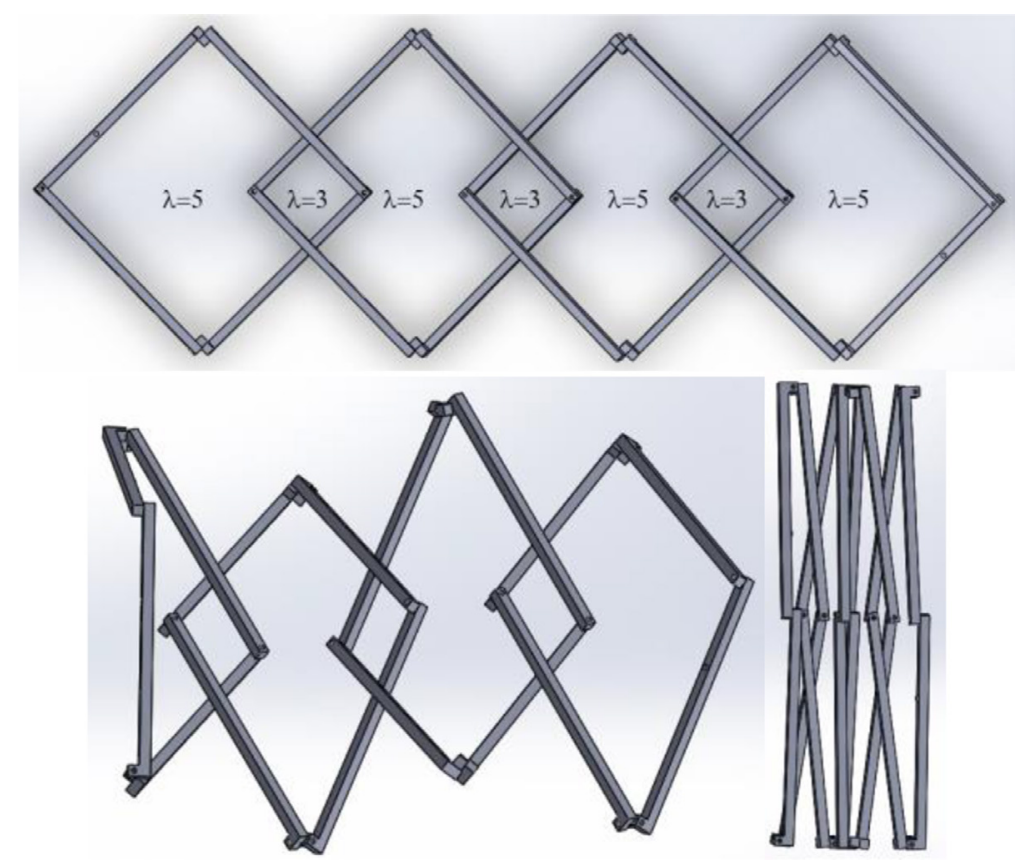

Figure 12: Deployment stages (deployed, semi-deployed and folded) of parallelogram loop version.

Fig. 12 shows CAD model of deployed, semi-deployed and folded configurations of parallelogram loop version.

\section{CONCLUSION}

This paper presents a systematic derivation method for assembling several identical single-loop Altmann linkages into deployable networks. Modules are assembled with common links and joints or with extra $\mathrm{R}$ or $2 \mathrm{R}$ joints. Using these derivation methods, one can design 
complicated multi-loop deployable networks from single-loop basic Altmann linkage without altering the mobility and the foldability of the module.

\section{REFERENCES}

[1] Phillips, J., Freedom in Machinery, Cambridge University Press, 2006.

[2] Bennett, G.T., A new mechanism. Engineering, 76, pp. 777-778, 1903.

[3] Baker, E.J. \& Hu, M., On spatial networks of overconstrained linkages. Mechanism and Machine Theory, 21(5), pp. 427-437, 1986. http://dx.doi.org/10.1016/0094-114X(86)90091-1

[4] Chen, Y., Design of Structural Mechanisms, PhD thesis, St Hugh's college, University of Oxford, 2003.

[5] Melin, N.O., Application of Bennett Mechanisms to Long-Span Shelters, PhD thesis, Magdalen College, University of Oxford, 2004.

[6] Myard, F.E., Contribution à la géométrie des systèmes articulés. Societe Mathématiques de France, 59, pp. 183-210, 1931.

[7] Huang, H., Deng, Q. \& Li, B., Mobile assemblies of large deployable structures. Journal of Space Structures, 5(1), pp. 1-14, 2012.

[8] Sarrus, P.T., Note sur la transformation des mouvements rectilignes alternatifs, en mouvements circulaires et réciproquement. Académie des Sciences, 36, pp. 1036-1038, 1853.

[9] Calatrava, V.S., Zur Faltbarkeit von Fachwerken, PhD thesis, Eidgenoessischen Technischen Hochschule Zürich, 1981.

[10] Bouten, S., Transformable Structures and Their Architectural Application, Master's dissertation, Ghent University, 2015.

[11] Kiper, G. \& Söylemez, E., Obtaining new linkages from jitterbug-like polyhedral linkages. In Proceedings of the International Symposium of Mechanism and Machine Science, AzIFToMM, Izmir Institute of Technology, 2010.

[12] Bricard, R., Leçons de cinématique Tome II. Cinématique Appliquée, Gauthier-Villars, Paris, pp. 7-12, 1927.

[13] Huang, H., Li, B., Zhu, J. \& Qi, X., New family of Bricard-derived deployable mechanisms. Journal of Mechanisms and Robotics, 2016.

http://dx.doi.org/10.1115/1.4032119

[14] Cui, J., Huang, H., Li, B. \& Deng, Z., A novel surface deployable antenna structure based on special form of Bricard linkages. Advances in Reconfigurable Mechanisms and Robots I, pp. 783-792, 2012.

http://dx.doi.org/10.1007/978-1-4471-4141-9_70

[15] Altmann, F.G., Über raümliche sechsgliedrige Koppelgetriebe, Sonderdruck aus derZeifschrift des Vereines Deutscher Ingenieure, 96(8), pp. 245-249, 1954.

[16] Baker, J.E., A geometrico-algebraic exploration of Altmann's linkage. Mechanism and Machine Theory, 28(2), pp. 249-260, 1993. http://dx.doi.org/10.1016/0094-114X(93)90091-9

[17] Alizade, R., Structural synthesis of robot manipulators. In Proceedings of the International Symposium of Mechanism and Machine Science, AzCIFToMM, Izmir Institute of Technology, pp. 11-32, 2010. 